\title{
Precision Measurements of Ionization and Dissociation Energies by Extrapolation of Rydberg Series: From $\mathrm{H}_{2}$ to Larger Molecules
}

\author{
D. Sprecher§, M. Beyer, and F. Merkt* \\ §SCS-Metrohm Foundation Award for best oral presentation
}

\begin{abstract}
Recent experiments are reviewed which have led to the determination of the ionization and dissociation energies of molecular hydrogen with a precision of $0.0007 \mathrm{~cm}^{-1}(8 \mathrm{~mJ} / \mathrm{mol}$ or $20 \mathrm{MHz})$ using a procedure based on high-resolution spectroscopic measurements of high Rydberg states and the extrapolation of the Rydberg series to the ionization thresholds. Molecular hydrogen, with only two protons and two electrons, is the simplest molecule with which all aspects of a chemical bond, including electron correlation effects, can be studied. Highly precise values of its ionization and dissociation energies provide stringent tests of the precision of molecular quantum mechanics and of quantum-electrodynamics calculations in molecules. The comparison of experimental and theoretical values for these quantities enable one to quantify the contributions to a chemical bond that are neglected when making the Born-Oppenheimer approximation, i.e. adiabatic, nonadiabatic, relativistic, and radiative corrections. Ionization energies of a broad range of molecules can now be determined experimentally with high accuracy (i.e. about $\left.0.01 \mathrm{~cm}^{-1}\right)$. Calculations at similar accuracies are extremely challenging for systems containing more than two electrons. The combination of precision measurements of molecular ionization energies with highly accurate ab initio calculations has the potential to provide, in future, fully reliable sets of thermochemical quantities for gas-phase reactions.
\end{abstract}

Keywords: High-resolution spectroscopy · Molecular hydrogen · Rydberg states · Thermochemistry

\section{Rydberg States and Ionization Energies}

Atomic and molecular Rydberg states are electronic states that can be thought of as consisting of an excited electron orbiting at large distances around a positively charged ion core to which it is bound by the attractive Coulomb interaction between particles of opposite charges. The quantized energy levels of the electronic motion can be described, in good approximation, by Rydberg's empirical formula ${ }^{[1,2]}$

$$
h c \tilde{v}_{n l}=E_{\mathrm{I}}\left(\alpha^{=}\right)-\frac{h c R_{\mathrm{M}}}{\left(n-\delta_{l}\right)^{2}}
$$

In Eqn. (1), $\tilde{v}_{n l}$ represents the spectral position of the Rydberg state with the principal and orbital angular-momentum quantum numbers $n$ and $l$, respectively. $h$, $c, E_{\mathrm{I}}\left(\alpha^{+}\right), R_{\mathrm{M}}$, and $\delta_{l}$ are Planck's constant, the speed of light in vacuum, the ionization

\footnotetext{
${ }^{\star}$ Correspondence: Prof. Dr. F. Merkt Laboratory of Physical Chemistry ETH Zurich

Wolfgang-Pauli-Str. 10

$\mathrm{CH}-8093$ Zürich

Tel.: +4144632 4367

E-mail: feme@xuv.phys.chem.ethz.ch
}

energy associated with the formation of a singly-charged atomic or molecular cation in quantum state $\alpha^{+}$, the mass-dependent Rydberg constant and the quantum defect of states with angular momentum quantum

number $l$, respectively. Rydberg states of the same value of $l$ and $\alpha^{+}$form infinite series (see Fig. 1a) converging at $n=\infty$ to the series limit $E_{\mathrm{I}}\left(\alpha^{+}\right)$, which corresponds to the energetic position of the quantum state

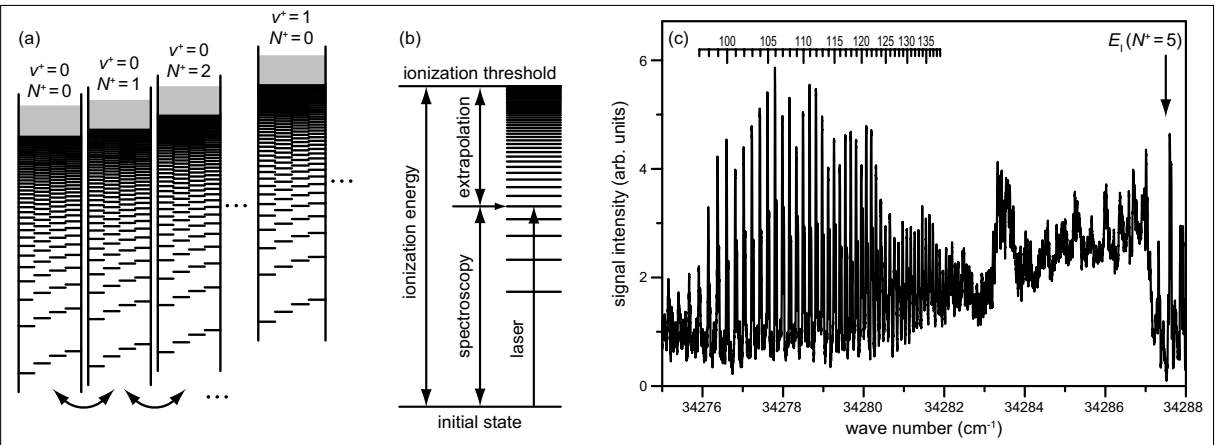

Fig. 1 (a) Schematic diagram of the energy level structure of molecular Rydberg states. Rydberg series of different / values converge to the ionization thresholds corresponding to each vibrational (labeled with the vibrational quantum number $\mathrm{V}^{+}$) and rotational $\left(\mathrm{N}^{+}\right)$energy levels of the molecular ion core. The bound states below the ionization thresholds are marked in black and the ionization continua above the thresholds in grey. (b) Determination of ionization energies by Rydberg-state spectroscopy. The transition frequencies from the ground state of the neutral molecule to successive Rydberg states of a series are measured. Extrapolation of the series using Rydberg's formula or multichannel quantum defect theory yields the position of the ionization threshold. (c) Selected section of the Rydberg spectrum of $\mathrm{He}_{2}$ displaying the $n p(l=1)$ series converging to the $N^{+}=5$ level of the $\mathrm{X}^{+2} \Sigma_{\mathrm{u}}^{+}\left(\mathrm{v}^{+}=0\right)$ ground state of the $\mathrm{He}_{2}{ }^{+}$ion measured following excitation from the $\mathrm{N}=5$ rotational level of the lowest-lying, metastable triplet a ${ }^{3} \sum_{\mathrm{u}}^{+}(\mathrm{v}=0)$ state of $\mathrm{He}_{2}$. The principal quantum numbers of the Rydberg states are given along the assignment bar, and the extrapolated position of the ionization threshold is indicated by the vertical arrow. 
$\alpha^{+}$of the ion core and marks the energetic onset of an ionization continuum. The infinite series of bound states with given values of $l$ and $\alpha^{+}$and the adjacent ionization continuum represent what is called an ionization channel.

In molecules, every electronic, vibrational, rotational and even hyperfine level of the ion core represents an ionization threshold, to which many Rydberg series converge, ${ }^{[3]}$ as depicted in Fig. 1a. The figure also sketches, in panel (b), how an ionization threshold can be determined by measuring the frequencies of transitions to Rydberg states and extrapolating the series to their limits. An actual measurement of a Rydberg series in $\mathrm{He}_{2}$ recorded from the metastable triplet a ${ }^{3} \sum_{u}^{+}(v=0)$ state is shown in panel (c). From this measurement, one can infer that the ionization threshold corresponding to the formation of the $\mathrm{v}^{+}=0, N^{+}=5$ rotational level of the $\mathrm{He}_{2}^{+} \mathrm{X}^{2} \sum_{\mathrm{u}}^{+}$ground electronic state is located $34287.582(6) \mathrm{cm}^{-1}$ above the $N=5$ rotational level of the metastable state.

At sufficiently large distance from the ion core, the potential experienced by the Rydberg electron becomes exactly Coulombic. Consequently, even large molecules have Rydberg states and these can be studied with the method illustrated in Fig. 1. Rydberg state extrapolation is therefore a general method to derive ionization energies.

Not only the adiabatic ionization energies $\left(E_{\mathrm{I}}^{(0)}\right)$ result from the analysis of Rydberg series in molecules, but also the relative positions of the vibrational, rotational and hyperfine quantum states of the ion with respect to those of the neutral molecule. $E_{\mathrm{I}}^{(0)}$, which is defined as the minimal energy required to produce the lowest quantum state of the ion from the lowest quantum state of the parent neutral molecule, ${ }^{[4]}$ represents a characteristic property of an atom or molecule, which can be used in the determination of thermochemical quantities such as standard 0 $\mathrm{K}$ reaction energies and enthalpies $\left(\Delta_{\mathrm{r}} U^{\ominus}\right.$ $\left.(0 \mathrm{~K})=\Delta_{\mathrm{r}} H^{\ominus}(0 \mathrm{~K})\right)$. For example, for the charge-transfer reaction $\mathrm{R}_{1}+\mathrm{R}_{2}^{+}=\mathrm{R}_{1}^{+}+$ $\mathrm{R}_{2}$, one finds

$$
\Delta_{\mathrm{r}} U^{\ominus}(0 \mathrm{~K})=E_{\mathrm{I}}^{(0)}\left(\mathrm{R}_{1}\right)-E_{\mathrm{I}}^{(0)}\left(\mathrm{R}_{2}\right)
$$

\section{Precision Measurements of Ionization and Dissociation Energies}

\subsection{Ionization Energies}

Ionization energies can be determined spectroscopically i) from photoabsorption or photoionization spectra by determining the onset of ionization continua, ${ }^{[5]}$ ii) from photoelectron spectra ${ }^{[6]}$ by measuring the kinetic energy of photoelectrons emitted in the ionization continuum following photoionization with a narrow-band radiation source, or iii) by Rydberg series extrapolation, as just explained. In the first case, the precision and accuracy of the measurements are limited by the fact that the onset of the ionization continua are never infinitely sharp, either because of stray electric fields or because the thresholds corresponding to the production of the cation in different rotational levels are not resolved, leaving ambiguities concerning the exact position of the ionization threshold. In the second case, the precision is limited by intrinsic difficulties associated with the measurements of photoelectron kinetic energies. Even when the detection is restricted to photoelectrons of near-zero kinetic energies, as in pulsed-field-ionization zerokinetic-energy (PFI-ZEKE) photoelectron spectroscopy, ${ }^{[7]}$ stray electric fields limit the accuracy to about $1 \mathrm{~cm}^{-1}$.

The advantage of Rydberg series extrapolation for the determination of ionization energies is that the precision is in principle only limited by the widths of the transitions observed in Rydberg spectra. Because the lifetimes of Rydberg states scale with $n^{3}$, the natural linewidths of high Rydberg states are small and the observed spectral linewidths are usually limited by the bandwidth of the radiation source used to record the spectra or by the Doppler effect. The determination of ionization energies by Rydberg series extrapolation has a long tradition in molecular physics. Classical examples are studies of the Rydberg spectrum of NO by Miescher and his coworkers ${ }^{[8]}\left(E_{\mathrm{I}}^{(0)}(\mathrm{NO})=\right.$ $\left.74721.5(5) \mathrm{cm}^{-1}\right)$, of $\mathrm{H}_{2}$ by Herzberg and Jungen $^{[9]}\left(E_{\mathrm{I}}^{(0)}\left(\mathrm{H}_{2}\right)=124417.2(4) \mathrm{cm}^{-1}\right)$, and of $\mathrm{N}_{2}$ by Huber and Jungen ${ }^{[10]}\left(E_{\mathrm{I}}^{(0)}\left(\mathrm{N}_{2}\right)\right.$ $=125667.032(65) \mathrm{cm}^{-1}$ ).

Rydberg extrapolation techniques have been combined with pulsed-field ionization techniques related to PFI-ZEKE photoelectron spectroscopy to obtain an even higher precision: Narrow-band pulsed lasers are used to resolve Rydberg series at high $n$ values $(n>100)$. Because such states are located less than $10 \mathrm{~cm}^{-1}$ below the ionization energies, the extrapolation with Rydberg's formula yields ionization energies $\left(E_{\mathrm{I}}\left(\alpha^{+}\right)\right.$in Eqn. (1)) accurate to better than $0.1 \mathrm{~cm}^{-1}$ and also information on the quantum defects $\left(\delta_{l}\right)$ and the rovibronic energy-level structure of the cations. Examples include the determination of the ionization energies of benzene ${ }^{[11]}$ and benzene-rare gas van der Waals complexes, ${ }^{[12]} \mathrm{N}_{2},{ }^{[13]} \mathrm{CO},{ }^{[14]}$ and $\mathrm{NH}_{3} \cdot{ }^{[15]}$ The advantage of this method is the straightforward analysis. Indeed, perturbations of the Rydberg series such as those discussed below in the context of Fig. 2 are small at high $n$ values and have no significant effect on the extrapolated values. However, systematic shifts of the high Rydberg states by stray electric fields, such as those caused by ions produced in the photoabsorption volume, rapidly increase with $n$ (as $n^{7}$ ) and are difficult to assess. Such shifts limit the absolute accuracy of the measurement of ionization energies to about $0.02 \mathrm{~cm}^{-1}$. [16]

The highest accuracy $\left(0.0007 \mathrm{~cm}^{-1}\right.$ and better) in the measurements of ionization energies is reached in measurements of Rydberg states with principal quantum numbers around $n=50 .[17,18]$ These states have principal quantum numbers low enough that they are not significantly affected by typical stray fields in the range of $1-10 \mathrm{mV} / \mathrm{cm}$ present in most spectrometers. Simultaneously, these quantum numbers are high enough for the Rydberg states to have long lifetimes and for uncertainties in the quantum-defect parameters not to have significant effects on the extrapolated values. In this range of $n$ values, perturbations of the Rydberg series can be significant and need to be rigorously taken into account. The arrows in Fig. 1a indicate that the series converging to different ionic states can perturb each other, which leads to deviations of the spectral positions from those predicted by Rydberg's formula (Eqn. (1)).

A generalization of Rydberg's formula which is able to treat these perturbations can be reached in the realm of multichannel quantum defect theory (MQDT), as described in detail in ref. [19]. This generalization necessitates the inclusion of additional parameters accounting for the perturbations. The effect of such parameters, which can be thought of as off-diagonal quantum defects, is illustrated in Fig. 2 for the simple case of two interacting series, as is encountered in the $\mathrm{p}$ series converging on the $N^{+}=0$ and $N^{+}=2$ rotational levels of the $\mathrm{X}^{+2} \sum_{\mathrm{g}}^{+}\left(\mathrm{v}^{+}=0\right)$ ground state of $\mathrm{H}_{2}^{+} \cdot[9,20]$ In the panels (a) and (b) of Fig. 2, the deviations between the spectral positions calculated with Rydberg's formula and those obtained for a weak (panel a, $\eta=0.04$, in quantum-defect units) and a strong (panel $b, \eta=0.2$ ) interaction parameter $\eta$ are plotted against the principal quantum number of the $N^{+}=0$ series in the range $n=50-100$. The deviations, and thus the effect of the series interaction, are strongest in the immediate vicinity of the positions of the Rydberg levels belonging to the $N^{+}=2$ series which have lower principal quantum numbers ( $n=23$ and 24) in this energetic range (see Fig. 2c). Because the series interactions are treated globally in the framework of MQDT, a single parameter $\left(\eta_{i j}\right)$ per pair of interacting series $(i, j)$ is often sufficient to describe the deviations of all Rydberg levels. The parameters used for the calculation of a Rydberg spectrum are 


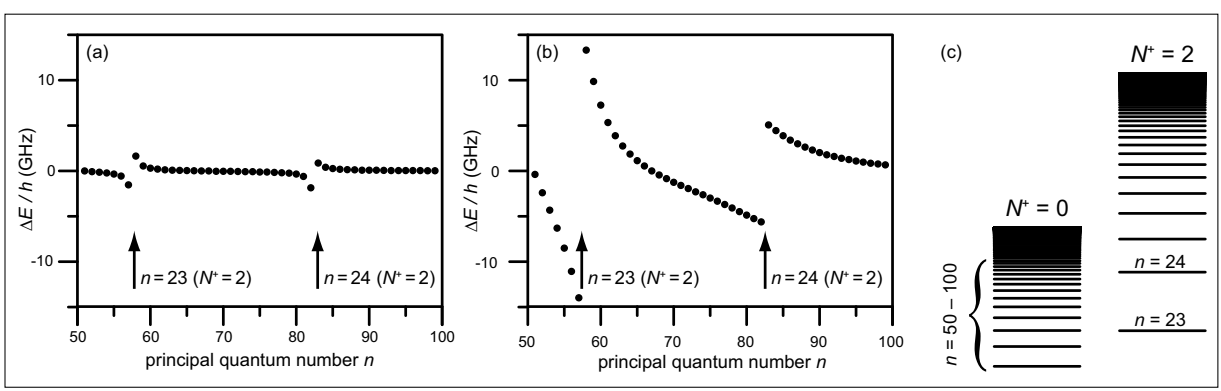

Fig. 2. a) and b): Model MQDT calculations illustrating the effects of an interaction between two Rydberg series converging on different rotational levels of a molecular cation assuming an interaction parameter $\eta=0.04$ (a) and $\eta=0.2$ (b). The relative positions of the series limits and the quantum defects correspond to the situation encountered for the singlet $p$ series converging on the $\mathrm{N}^{+}=0$ and $\mathrm{N}^{+}=2$ rotational levels of the $\mathrm{X}^{+}{ }^{2} \Sigma_{\mathrm{g}}^{+}\left(\mathrm{v}^{+}=0\right)$ ground state of $\mathrm{H}_{2}{ }^{+}$. The results of the calculations are presented as deviations of the Rydberg levels of the $N^{+}=0$ series from the positions resulting from Rydberg's formula as a function of their principal quantum number. These deviations are strongest near the positions of the $n=23$ and $n=24$ members of the $N^{+}=2$ series. c) Schematic energy level diagram of the relevant series.

the ionization energies $E_{\mathrm{I}}\left(\alpha^{+}\right)$, the quantum defects $\delta_{l}$, and the interaction parameters $\eta_{i j}$ coupling the different series. These parameters can be determined either ab initio (see ref. [19]) or in a fit to experimental data. The results presented in the next section demonstrate that ionization energies can be determined with a precision of 1 $\mathrm{MHz}\left(0.00003 \mathrm{~cm}^{-1}\right)$ when series interactions are considered, which is more than two orders of magnitude more precise than by extrapolation with Rydberg's formula.

\subsection{Determination of Dissociation Energies}

Measurements of dissociation energies $D_{0}(\mathrm{R}-\mathrm{A})$, defined as the minimal energy required to dissociate the molecules in their ground state to form the fragments $\mathrm{R}$ and $\mathrm{A}$ in their ground states, are more difficult because transitions to the dissociation continua are usually very weak and the continua have diffuse onsets. Moreover, potential energy barriers along the dissociation coordinate can prevent the observation of the dissociation products at threshold. The determination of the dissociation thresholds by extrapolation of the measured positions of bound levels is not as easy as for ionization thresholds because the long-range part of the potentials is not Coulombic and does not, in general, support infinite series of bound levels.

In diatomic molecules, direct potential fits to experimental vibrational and rotational level positions can, in favorable cases, provide energies accurate to about $1 \mathrm{~cm}^{-1}$ or, in some cases, even better. ${ }^{[21]}$ In polyatomic molecules, such fits are not straightforward and rarely lead to uncertainties in the dissociation energies smaller than a few $\mathrm{cm}^{-1}$. In some cases, very precise dissociation energies can be derived from the observation of the onset of predissociation, as was demonstrated for $\mathrm{H}_{2} \mathrm{O}$, for which the $\mathrm{O}-\mathrm{H}$ bond dissociation energy $D_{0}(\mathrm{HO}-\mathrm{H})$ was determined to

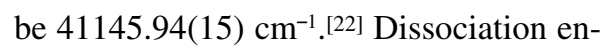
ergies can also be used to derive standard 0 $\mathrm{K}$ reaction energies and enthalpies. For instance, the reaction energy of the reaction $R_{1}-A+R_{2}=R_{1}+R_{2}-A$ can be determined using the relation

$$
\Delta_{\mathrm{r}} \mathrm{U}^{\ominus}(0 \mathrm{~K})=D_{0}\left(\mathrm{R}_{1}-\mathrm{A}\right)-D_{0}\left(\mathrm{R}_{2}-\mathrm{A}\right)
$$

$0 \mathrm{~K}$ reaction enthalpies for a wide range of gas-phase reactions can be derived from ionization and dissociation energies by combining equations such as Eqns. (2) and (3) in larger thermodynamical cycles.

Dissociation and ionization energies are related by the positive-ion thermodynamical cycle: ${ }^{[23]}$

$$
\begin{aligned}
E_{\mathrm{I}}^{(0)}(\mathrm{R}-\mathrm{A})= & E_{\mathrm{I}}^{(0)}(\mathrm{A})+D_{0}(\mathrm{R}-\mathrm{A})- \\
& D_{0}\left(\mathrm{R}-\mathrm{A}^{+}\right),
\end{aligned}
$$

where $E_{\mathrm{I}}^{(0)}(\mathrm{R}-\mathrm{A})$ and $E_{\mathrm{I}}^{(0)}(\mathrm{A})$ designate the adiabatic ionization energies of R-A and A, and $D_{0}(\mathrm{R}-\mathrm{A})$ and $D_{0}\left(\mathrm{R}-\mathrm{A}^{+}\right)$the dissociation energies of R-A and R-A ${ }^{+}$, respectively. In general, Eqn. (4) cannot be used to derive precise values of dissociation energies, even if the ionization energies of R-A and A are precisely known. Molecular hydrogen is an exception. $\mathrm{H}_{2}^{+}$being a one-electron system, its dissociation energy can be calculated $a b$ initio with almost arbitrary precision $\left(D_{0}\left(\mathrm{H}_{2}^{+}\right)=21379.35024(6) \mathrm{cm}^{-1}[24]\right)$. A measurement of the ionization energy of $\mathrm{H}_{2}$ thus also yields a value of $D_{0}\left(\mathrm{H}_{2}\right)$ by using Eqn. (4).

\section{The lonization and Dissociation Energies of Molecular Hydrogen}

To derive the ionization and dissociation energies of $\mathrm{H}_{2}, \mathrm{HD}$, and $\mathrm{D}_{2}$ with high accuracy, we determined their ionization energies by Rydberg-series extrapolation and their dissociation energies using Eqn. (4). The ionization energy of the hydrogen atom $\left(E_{\mathrm{I}}(\mathrm{H})=109678.77174 \mathrm{~cm}^{-1}\right.$ and $\left.E_{\mathrm{I}}(\mathrm{D})=109708.61455 \mathrm{~cm}^{-1}[25]\right)$, and the dissociation energies of $\mathrm{H}_{2}^{+}, \mathrm{HD}^{+}$, and $\mathrm{D}_{2}^{+[24]}$ are precisely known. The ionization energy of molecular hydrogen was obtained as a sum of three energy intervals measured in separate experiments, the first from the $\mathrm{X}^{1} \sum_{\mathrm{g}}^{+}(\mathrm{v}=0, N=0$ or 1$)$ levels to the $\mathrm{EF}^{1} \sum_{\mathrm{g}}^{+}(\mathrm{v}=0, N=0$ or 1$)$ levels by twophoton Doppler-free spectroscopy, ${ }^{[26]}$ the second between the $\mathrm{EF}^{1} \sum_{\mathrm{g}}^{+}(\mathrm{v}=0, N=0$ or 1$)$ levels and $n \mathrm{p}$ Rydberg states with $n$ values around 50 belonging to series converging on the $\mathrm{X}^{+2} \sum_{\mathrm{g}}^{+}\left(\mathrm{v}^{+}=0, N^{+}=0\right.$ or 1$)$ of the ion by UV laser spectroscopy, ${ }^{[18,27,28]}$ and the third between high the $n \mathrm{p}$ Rydberg states and their series limits by millimeter-wave spectroscopy and MQDT. ${ }^{[17,28,29]}$

As illustration of these measurements, Fig. 3 shows a spectrum of the p Rydberg states of HD located below the $\mathrm{X}^{+}\left(\mathrm{v}^{+}=0\right.$, $N^{+}=0$ ) ground state of $\mathrm{HD}^{+}$recorded from the $\mathrm{EF}(\mathrm{v}=0, N=0)$ state. The observed Rydberg series interacts strongly with the

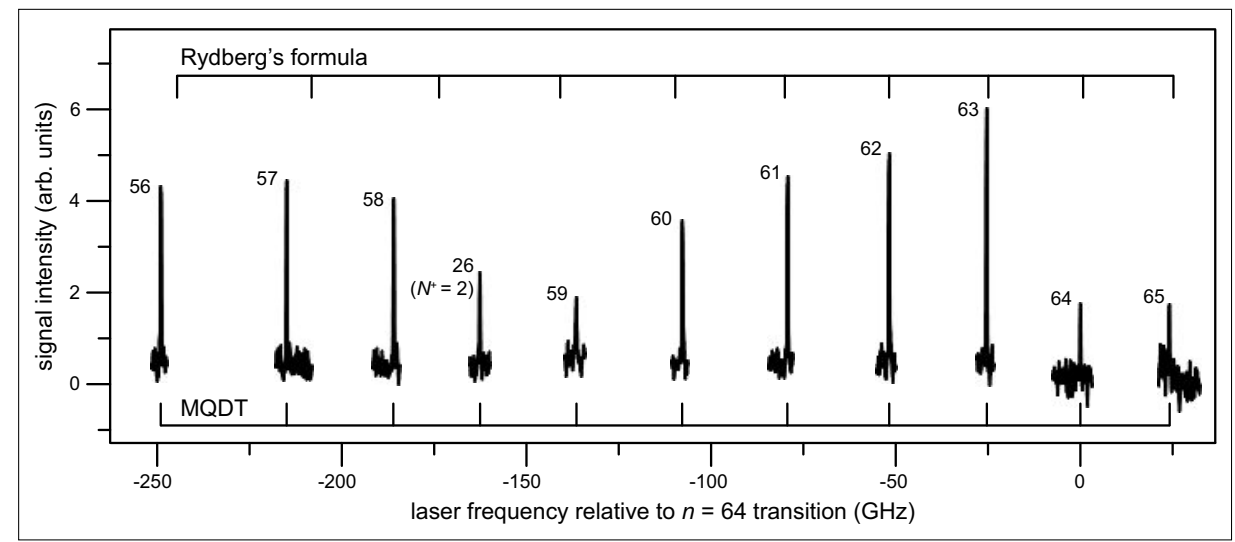

Fig. 3. Spectrum of the $n=56-65 p$ Rydberg series of HD converging to the $N^{+}=0, v^{+}=0$ level of the electronic ground state of $\mathrm{HD}^{+}$recorded from the $\mathrm{EF}(\mathrm{v}=0, \mathrm{~N}=0)$ state. The perturbation caused by the interaction with the $n=26 \mathrm{p}$ Rydberg state with a $N^{+}=2$ ion core can be recognized from strong deviations from the positions predicted with Rydberg's formula (upper assignment bar). The positions calculated using MQDT are given along the lower assignment bar. 
p Rydberg series converging to the $N^{+}=2$ state in the way illustrated by Fig. 2. While Rydberg's formula fails to reproduce the observed rotational interactions (see upper assignment bar), the MQDT calculations are in agreement with the observed positions within the experimental uncertainty (see lower assignment bar in Fig. 3). After taking into account all known sources of systematic errors, such dc and ac Stark shifts, pressure shifts, Doppler shifts, and frequency chirps of the pulsed laser, the adiabatic ionization energy of HD could be determined with an uncertainty of 11 $\mathrm{MHz}\left(E_{\mathrm{I}}^{(0)}(\mathrm{HD})=124568.48581(36) \mathrm{cm}^{-1}\right.$, which led to a dissociation energy $\mathrm{D}_{0}(\mathrm{HD})$ of $36405.78366(36) \mathrm{cm}^{-1}[28]$ ).

The main factor limiting the precision and accuracy of these measurements are Stark shifts caused by ions generated in the measurement volume. When the measurements are carried out through the EF intermediate state, these ions are unavoidable, because the efficient driving of the EF - X two-photon transition requires high laser intensities at a frequency sufficiently high to directly ionize the EF state in a singlephoton process.

In a new series of measurements, the GK state instead of the EF state was used as intermediate state for the excitation of high Rydberg states, ${ }^{[30]}$ with the goal of avoiding the generation of ions in the experimental volume. Compared to the EF state, the GK state lies higher in energy and can be excited efficiently (and thus with lower laser intensities) from the ground state in a resonant two-photon transition. Sections of the observed spectrum in ortho- $\mathrm{H}_{2}$ are depicted in Fig. 4. The reduced transition frequency and the improved collimation of the molecular beam enabled the reduction of the Doppler width of the observed lines to about $20 \mathrm{MHz}$ (for comparison, the line widths in Fig. 3 are about $120 \mathrm{MHz}$ because of the Doppler effect and inhomogeneous broadening by the stray fields). No rotational series perturbations of the kind illustrated in Fig. 2 occur in the spectral range shown in Fig. 4. Nevertheless, the observed Rydberg series cannot be accurately described by Rydberg's formula. The reason for the deviations are perturbations caused by the hyperfine interaction in Rydberg series converging to different hyperfine components of the $\mathrm{v}^{+}=0, N^{+}=1$ ground state of ortho- $\mathrm{H}_{2}^{+}$. An extension of MQDT that includes hyperfine effects ${ }^{[17,31]}$ had to be employed to reproduce the observed perturbations within the experimental uncertainties.

In this way, the ionization energy of $\mathrm{H}_{2}$ from the $\mathrm{GK}(\mathrm{v}=1, N=1)$ level could be determined with an absolute accuracy of 1.2 MHz, which sets new standards in laser spectroscopic investigations of molecular Rydberg states.

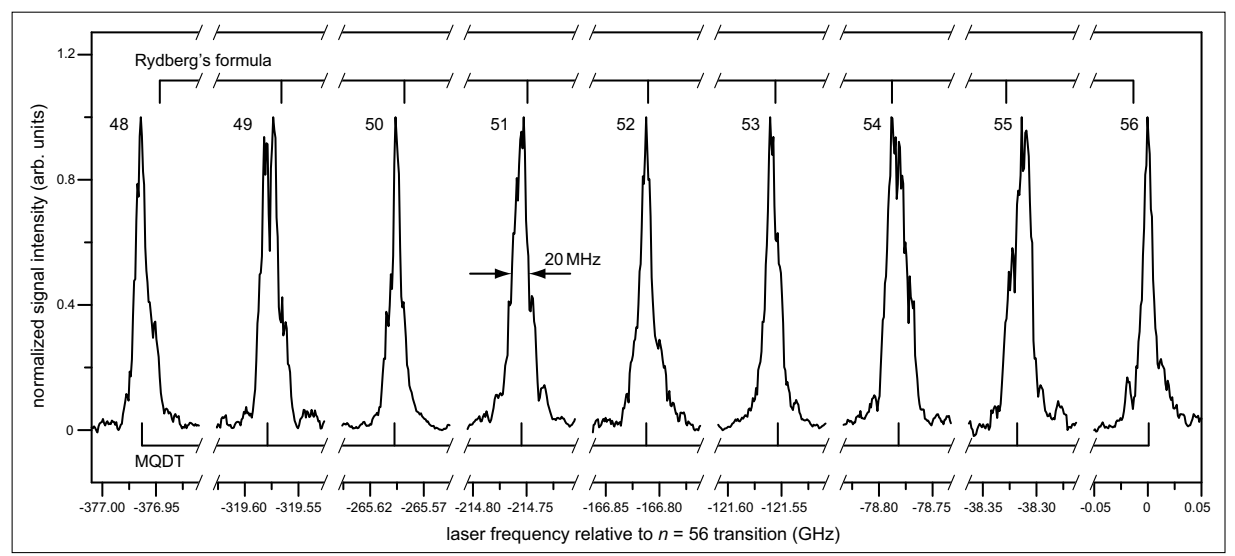

Fig. 4. Intensity-normalized sections of the spectrum of the $\mathrm{p}(\mathrm{N}=1)$ Rydberg series of ortho- $\mathrm{H}_{2}$ converging to the $N^{+}=1, \mathrm{~V}^{+}=0$ state of the electronic ground state of ortho- $\mathrm{H}_{2}^{+}$recorded from the GK $(v=1, N=1)$ state. Next to each line the principal quantum number $n$ of the corresponding Rydberg state is given. The predictions using Rydberg's formula and MQDT are given above and below the spectra, respectively.

\section{Discussion and Conclusions}

\subsection{The Dissociation Energy of Molecular Hydrogen}

Simultaneously to, and independently of, our measurements, the ionization and dissociation energies of $\mathrm{H}_{2}, \mathrm{HD}$, and $\mathrm{D}_{2}$ were also determined in $a b$ initio calculations incorporating the adiabatic and nonadiabatic corrections to the Born-Oppenheimer energies and also the relativistic, radiative, and finite-nuclear-size corrections. ${ }^{[32,33]}$ The magnitude of these correction terms for the case of the dissociation energy are listed in Table I for all three isotopomers. The adiabatic and nonadiabatic corrections take the coupling between the nuclear and electronic motions into account. Both correction terms scale with the ratio between the electron mass and the nuclear reduced mass and are therefore largest for $\mathrm{H}_{2}$. The relativistic correction originates from the velocity-dependent mass of the electrons and its magnitude is very similar for all isotopomers. The same is true for the radiative corrections, which have their origin in the interaction of the electron with its own electromagnetic field. The finite-nuclearsize correction scales with the size of the nuclei and is the smallest of all determined corrections.

At present, experimental and theoretical values agree within their combined uncertainties of $0.001 \mathrm{~cm}^{-1}(30 \mathrm{MHz})$. This agreement validates the theoretical values of the correction terms listed in Table I. The comparison also shows, among others, that it is necessary to include the electronpositron continuum (as part of the radiative correction) to account for our experimental results. Measurements of energy intervals in molecules at high precision indeed contain information on interactions of relevance in high-energy physics. ${ }^{[34]}$

\subsection{Accurate Thermochemistry}

Thermochemical networks are essential tools to describe and assess chemical equilibria in systems of coupled gas-phase reactions, for instance in combustion, atmospheric chemistry and for certain classes of astrophysical problems. With such networks, available thermochemical data on simple systems, properly weighted

Table I. Results of $a b$ initio calculations ${ }^{[32,33]}$ of the dissociation energies of $H_{2}, H D$, and $D_{2}$ and comparison with experimental values. ${ }^{[18,27,28]}$ The $a b$ initio values are determined as sums of corrections of different origin to the values obtained within the Born-Oppenheimer approximation. All values are given in units of $\mathrm{cm}^{-1}$.

\section{Born-Oppenheimer}

Adiabatic correction

Nonadiabatic correction

Relativistic correction

Radiative correction

Finite-nuclear-size correction

Total theory

Experiment

$$
D_{0}\left(\mathrm{H}_{2}\right)
$$

36112.5927(1)

5.7711(1)

$0.4339(2)$

$-0.5319(3)$

$-0.1964(8)$

$0.0000(1)$

36118.0695(10)

36118.0696(4)

$$
D_{0}(\mathrm{HD})
$$

36401.9332(1)

$$
\text { 4.2509(1) }
$$$$
0.3267(2)
$$

$36405.7828(10)$

36405.7837(4)

$$
\begin{gathered}
D_{0}\left(\mathrm{D}_{2}\right) \\
36746.1623(1) \\
2.7725(1)
\end{gathered}
$$

36748.3633(9)

36748.3629(7) 
by their uncertainties, are used to derive thermodynamical properties of progressively more complex molecular systems in a systematic and optimal manner, on the basis of systems of equations of the type of Eqns. (2)-(4). ${ }^{[23]}$ Redundancy of the data can be exploited to detect problems with the input values, and $a b$ initio quantum chemistry can be used to check suspect data or to improve missing links in the overall thermochemical network.

Though remarkably successful, this procedure occasionally suffers from the facts that i) experimental uncertainties are difficult to assess, ii) available input data from experiment or calculations occasionally have too large uncertainties to be of use for a given reaction, which tends to be the case when they exceed the thermal energy $(k T)$ at the temperature of interest, and iii) error propagation in large thermochemical networks can lead, for desired thermochemical properties, to uncertainties larger than $k T$ at the temperature where these properties are needed, even if all input data have uncertainties below $k T$. The possibility that the thermochemical network fails for a specific reaction system can therefore not always be ruled out.

The determination of ionization and dissociation energies with such low uncertainties that they are entirely negligible, even at low temperature, say $10 \mathrm{~K}$ where $k T=1.38 \cdot 10^{-22} \mathrm{~J}$ (or $R T \approx 80 \mathrm{~J} / \mathrm{mol}$ or $k T /$ $\left.h c \approx 7 \mathrm{~cm}^{-1}\right)$, represents a possible way to improve thermochemical data. Taking into account the possibility of error propagation in large thermochemical networks, one may desire ionization and dissociation energies to be known with an accuracy 100 times higher, i.e. $0.07 \mathrm{~cm}^{-1}$. This accuracy is currently out of reach of measurements of bond-dissociation energies and much higher than can be achieved by $a b$ initio quantum chemistry in the realm of the Born-Oppenheimer approximation, at least for molecules containing light atoms. The determination of ionization energies by Rydberg series extrapolation can be achieved with the requested accuracy for an increasing range of molecular systems (see Section 2). However, they are demanding.

An approach to accurate thermochemistry based on precision measurements, if at all practicable, would necessitate a combination of experiment and theory, with the goal of mutual validation of experimental and theoretical methods in progressively more complex molecular systems. Because one may expect a calculation that successfully predicts an ionization energy with high accuracy to also be able to predict a dissociation energy with the same accuracy, the validation by experiment only needs to be made for ionization energies. On the experimental side, this approach would need systematic studies of Rydberg states in larger molecules and the exploitation of multiphoton excitation sequences designed to access nonpenetrating Rydberg states which are long lived and not affected by perturbations. On the theory side, this approach would need conceptual advances, in particular in the development of standard procedures to evaluate adiabatic, nonadiabatic, relativistic and radiative corrections to energies calculated using the Born-Oppenheimer approximation.

The current progress in high-resolution spectroscopy, the rapid increase of computing resources and recent developments i) in the calculation of adiabatic, nonadiabatic and relativistic corrections to molecular energies, ${ }^{[35,36]}$ ii) in the determination of zero-point vibrational energies, ${ }^{[37]}$ and iii) in relativistic quantum chemistry ${ }^{[38,39]}$ justifies some (prudent) optimism. In this context, the results presented here for $\mathrm{H}_{2}$ show that both theoretical and experimental determination of ionization and dissociation energies in two-electron systems can reach an accuracy better than $0.001 \mathrm{~cm}^{-1}$, which amply satisfies the accuracy criterion mentioned above. The experimental data on $\mathrm{He}_{2}$ and $\mathrm{He}_{2}^{+}$shown in Fig. 1c provide reference energies against which calculations for three and four electron systems may be compared in future.

\section{Acknowledgments}

This work is supported financially by the Swiss National Science Foundation under Project 200020-135342 and by the European Research Council advanced grant program under Project 228286.

Received: March 4, 2013

[1] J. R. Rydberg, Z. Phys. Chem. 1890, 5, 227.

[2] T. F. Gallagher, 'Rydberg Atoms', Cambridge University Press, Cambridge, 1994.

[3] F. Merkt, Ann. Rev. Phys. Chem. 1997, 48, 675.

[4] K. P. Huber, G. Herzberg, 'Molecular Spectra and Molecular Structure', Vol. IV, 'Constants of diatomic molecules', Van Nostrand Reinhold, New York, 1979

[5] J. Berkowitz, 'Photoabsorption, Photoionization and Photoelectron Spectroscopy', Academic Press, New York, 1979

[6] D. W. Turner, C. Baker, A. D. Baker, C. R. Brundle, 'Molecular Photoelectron Spectroscopy: A Handbook of $\mathrm{He} 584 \AA$ Spectra', John Wiley \& Sons, New York, 1970.

[7] K. Müller-Dethlefs, E. W. Schlag, Ann. Rev. Phys. Chem. 1991, 42, 109.

[8] E. Miescher, Can. J. Phys. 1976, 54, 2074.
[9] G. Herzberg. C. Jungen, J. Mol. Spec. 1972, 41, 425.

[10] K. P. Huber, C. Jungen, J. Chem. Phys. 1990, $92,850$.

[11] R. G. Neuhauser, K. Siglow, H. J. Neusser, $J$. Chem. Phys. 1997, 106, 896.

[12] K. Siglow, R. Neuhauser, H. J. Neusser, $J$. Chem. Phys. 1999, 110, 5589

[13] R. Seiler, U. Hollenstein, G. M. Greetham, F. Merkt, Chem. Phys. Lett. 2001, 346, 201.

[14] U. Hollenstein, R. Seiler, A. Osterwalder, M. Sommavilla, A. Wüest, P. Rupper, S. Willitsch, G. M. Greetham, B. Brupbacher-Gatehouse, F. Merkt, Chimia 2001, 55, 759 .

[15] R. Seiler, U. Hollenstein, T. P. Softley, F. Merkt, J. Chem. Phys. 2003, 118, 10024.

[16] A. Osterwalder, S. Willitsch, F. Merkt, J. Mol. Struct. 2001, 599, 163.

[17] A. Osterwalder, A. Wüest, F. Merkt, C. Jungen, J. Chem. Phys. 2004, 121, 11810.

[18] J. Liu, E. J. Salumbides, U. Hollenstein, J. C. J. Koelemeij, K. S. E. Eikema, W. Ubachs, F. Merkt, J. Chem. Phys. 2009, 130, 174306.

[19] C. Jungen, 'Elements of quantum defect theory', in 'Handbook of High-resolution Spectroscopy', Eds. M. Quack, F. Merkt, Vol. 1, pp 471-510, John Wiley \& Sons, Chichester, 2011.

[20] U. Fano, Phys. Rev. A 1970, 2, 353

[21] R. J. Le Roy, C. C. Haugen, J. Tao, H. Li, Mol. Phys. 2011, 109, 435

[22] P. Maksyutenko, T. R. Rizzo, O. V. Boyarkin, J. Chem. Phys. 2006, 125, 181101.

[23] B. Ruscic, R. E. Pinzon, G. von Laszewski, D. Kodeboyina, A. Burcat, D. Leahy, D. Montoya, A. F. Wagner, J. Phys.: Conf. Ser. 2005, 16, 561 .

[24] V. I. Korobov, Phys. Rev. A 2008, 77, 022509.

[25] J. Mohr, B. N. Taylor, D. B. Newell, Rev. Mod. Phys. 2008, 80, 633.

[26] S. Hannemann, E. J. Salumbides, S. Witte, R. T. Zinkstok, E.-J. van Duijn, K. S. E. Eikema, W. Ubachs, Phys. Rev. A 2006, 74, 062514.

[27] J. Liu, D. Sprecher, C. Jungen, W. Ubachs, F. Merkt, J. Chem. Phys. 2010, 132, 154301.

[28] D. Sprecher, J. Liu, C. Jungen, W. Ubachs, F. Merkt, J. Chem. Phys. 2010, 133, 111102.

[29] H. A. Cruse, C. Jungen, F. Merkt, Phys. Rev. A 2008, 77, 042502.

[30] D. Sprecher, M. Beyer, F. Merkt, Mol. Phys. accepted for publication.

[31] H. J. Wörner, U. Hollenstein, F. Merkt, Phys. Rev. A 2003, 68, 032510.

[32] K. Piszczatowski, G. Łach, M. Przybytek, J. Komasa, K. Pachucki, B. Jeziorski, J. Chem. Theory Comput. 2009, 5, 3039.

[33] K. Pachucki, J. Komasa, Phys. Chem. Chem. Phys. 2010, 12, 9188

[34] M. Quack, Faraday Discuss. 2011, 150, 533.

[35] Y. J. Bomble, J. Vázquez, M. Kállay, C. Michauk, P. G. Szalay, A. G. Császár, J. Gauss, J. F. Stanton, J. Chem. Phys. 2006, 125, 064108.

[36] K. A. Peterson, D. Feller, D. A. Dixon, Theor. Chem. Acc. 2012, 131, 1079.

[37] S. Manzhos, T. Carrington Jr, K. Yamashita, $J$. Phys. Chem. Lett. 2011, 2, 2193.

[38] W. Kutzelnigg, Chem. Phys. 2012, 395, 16.

[39] M. Reiher, A. Wolf, 'Relativistic Quantum Chemistry - The Fundamental Theory of Molecular Science', Wiley-VCH, 2009. 